\title{
Insights into open/closed conformations of the catalytically active human guanylate kinase as investigated by small-angle $X$-ray scattering
}

\author{
Rohit Jain $^{1}$ - Nazimuddin Khan ${ }^{1} \cdot$ Andreas Menzel $^{2}$ - Ivan Rajkovic ${ }^{2}$. \\ Manfred Konrad $^{1} \cdot$ Simone Techert ${ }^{1,3,4}$
}

Received: 16 July 2015 / Accepted: 31 August 2015 / Published online: 7 October 2015

(C) The Author(s) 2015. This article is published with open access at Springerlink.com

\begin{abstract}
Bio-catalysis is the outcome of a subtle interplay between internal motions in enzymes and chemical kinetics. Small-angle X-ray scattering (SAXS) investigation of an enzyme's internal motions during catalysis offers an integral view of the protein's structural plasticity, dynamics, and function, which is useful for understanding allosteric effects and developing novel medicines. Guanylate kinase (GMPK) is an essential enzyme involved in the guanine nucleotide metabolism of unicellular and multicellular organisms. It is also required for the intracellular activation of numerous antiviral and anticancer purine nucleoside analog prodrugs. Catalytically active recombinant human GMPK (hGMPK) was purified for the first time and changes in the size and shape of open/closed hGMPK were tracked by SAXS. The binding of substrates (GMP + AMPPNP or Ap5G or GMP + ADP) resulted in the compaction of size and shape of hGMPK. The structural changes between open and completely closed hGMPK
\end{abstract}

R. Jain and N. Khan contributed equally to this work.

Electronic supplementary material The online version of this article (doi:10.1007/s00249-015-1079-9) contains supplementary material, which is available to authorized users.

\section{Simone Techert}

stecher@mpibpc.mpg.de; simone.techert@desy.de

Manfred Konrad

mkonrad@mpibpc.mpg.de

1 Max Planck Institute for Biophysical Chemistry, Am Fassberg 11, 37077 Göttingen, Germany

2 Paul Scherrer Institute, 5232 Villingen, Switzerland

3 Institute for X-ray Physics at University of Göttingen, Friedrich-Hund-Platz 1, 37077 Göttingen, Germany

4 FS-SCS at DESY, Notkestraße 85, 22607 Hamburg, Germany conformation were confirmed by observing differences in the hGMPK secondary structures with circular dichroism spectroscopy.

Graphical abstract

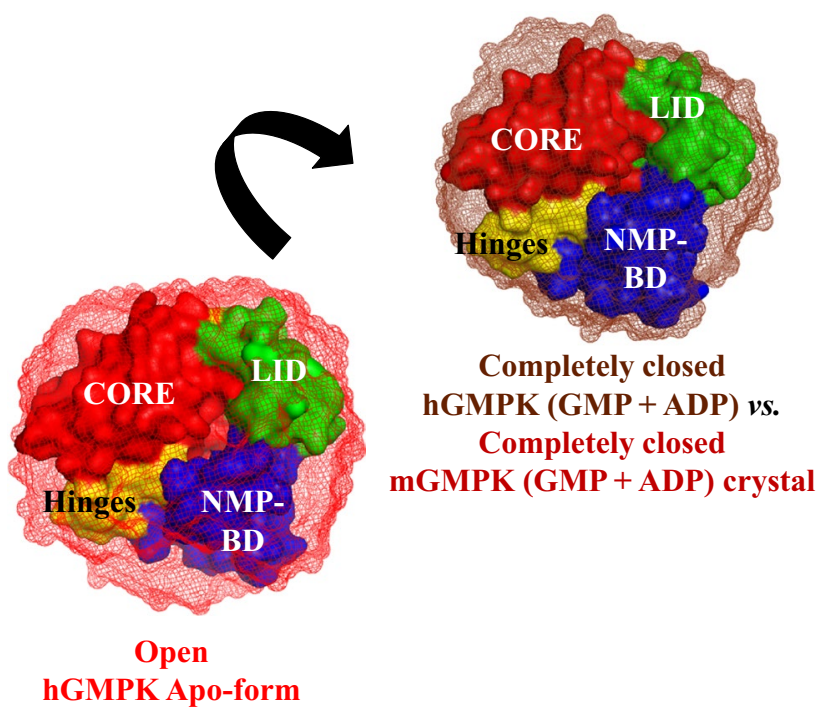

Keywords Protein conformations · Enzyme - Guanylate kinase $($ GMPK) $\cdot$ Nucleotide kinase $\cdot$ Small-angle X-ray scattering (SAXS)

$\begin{array}{ll}\begin{array}{l}\text { Abbreviations } \\ \text { ADP }\end{array} & \text { Adenosine 5'-diphosphate } \\ \text { AKs, AMP kinases } & \text { Adenylate kinases } \\ \text { AMP-PNP } & \begin{array}{l}\text { Adenosine } 5^{\prime}-(\beta, \gamma \text {-imido) triphos- } \\ \text { phate, non-hydrolysable ATP analog }\end{array} \\ \text { Ap5G } & \mathrm{P}^{1}-\left(5^{\prime} \text {-Adenosyl) } \mathrm{P}^{5}-\left(5^{\prime} \text {-guanosyl }\right)\right. \\ \text { pentaphosphate, non-hydrolysable } \\ \text { bi-substrate analog }\end{array}$




\begin{tabular}{|c|c|}
\hline cGMP & $\begin{array}{l}\text { 3', 5' -cyclic guanosine } \\
\text { monophosphate }\end{array}$ \\
\hline DTT & Dithiothreitol \\
\hline GMP & $\begin{array}{l}\text { Guanosine } 5 \\
\text {-mononhosphate }\end{array}$ \\
\hline GDP & Guanosine 5' -diphosphate \\
\hline GTP & Guanosine 5' -triphosphate \\
\hline HEPES & $\begin{array}{l}\text { 4-(2-hydroxyethyl)pipera- } \\
\text { zine-1-ethanesulfonic acid }\end{array}$ \\
\hline hGMPK & Human guanylate kinase \\
\hline hGMPK (apo) & $\begin{array}{l}\text { Open hGMPK form } \\
\text { (ligand-free) }\end{array}$ \\
\hline hGMPK (GMP + AMP-PNP) & $\begin{array}{l}\text { Completely closed hGMPK } \\
\text { form } 1\end{array}$ \\
\hline hGMPK (Ap5G) & $\begin{array}{l}\text { Completely closed hGMPK } \\
\text { form } 2\end{array}$ \\
\hline hGMPK (GMP + ADP) & $\begin{array}{l}\text { Completely closed hGMPK } \\
\text { form } 3\end{array}$ \\
\hline mGMPK & Mouse guanylate kinase \\
\hline NMP kinases, NMPKs & $\begin{array}{l}\text { Nucleoside monophos- } \\
\text { phate kinases }\end{array}$ \\
\hline NMP-BD & NMP binding domain \\
\hline PSI & Paul Scherrer Institute \\
\hline SAXS & $\begin{array}{l}\text { Small-angle X-ray } \\
\text { scattering }\end{array}$ \\
\hline SDS-PAGE & $\begin{array}{l}\text { Sodium dodecyl sul- } \\
\text { fate-Polyacrylamide gel } \\
\text { electrophoresis }\end{array}$ \\
\hline SLS & Swiss Light Source \\
\hline SUMO & $\begin{array}{l}\text { Small ubiquitin-related } \\
\text { modifier }\end{array}$ \\
\hline yGMPK & Yeast guanylate kinase \\
\hline \multicolumn{2}{|l|}{ List of symbols } \\
\hline$D_{\max }$ & Maximum dimensions \\
\hline$I(q)$ & X-ray scattering intensity \\
\hline$I(0)$ & Forward scattered intensity \\
\hline$P(r)$ & $\begin{array}{l}\text { Pair distance distribution } \\
\text { function }\end{array}$ \\
\hline$P d b$ & $\begin{array}{l}\text { Protein Data Bank file } \\
\text { format }\end{array}$ \\
\hline $\mathrm{pH} / \mathrm{pH}$ scale & $\begin{array}{l}\text { Hydronium ion concentra- } \\
\text { tion measurement }\end{array}$ \\
\hline & Momentum transfer \\
\hline$R_{\mathrm{g}}$ & Radius of gyration \\
\hline
\end{tabular}

\section{Introduction}

Guanylate kinase (GMPK, ATP:GMP phosphotransferase, EC 2.7.4.8) is an essential enzyme involved in the guanine nucleotide metabolism of unicellular and multicellular organisms. It catalyzes the reversible phosphoryl group transfer from ATP to GMP yielding GDP and ADP (Beck et al. 2003; Choi and Zocchi 2007; Sekulic et al. 2002). GMPK plays an important role in the recycling of the secondary messenger cGMP and thereby regulates the supply of guanine nucleotides to various signal transduction pathways (Hall and Kuhn 1986; Konrad 1992). In addition to its physiological roles, GMPK is also required for the intracellular activation of numerous antiviral and anticancer purine nucleoside analog prodrugs (Sekulic et al. 2002). Prominent examples are 6-thioguanine and 6-mercaptopurine, which are used for the treatment of acute lymphoblastic leukemia and as immunosuppressive agents, respectively (Ardiani et al. 2009; Karran 2006; Karran and Attard 2008; Zhang et al. 2013). Moreover, efficient agents routinely used for the treatment of herpes infections, such as ganciclovir and acyclovir, are first phosphorylated by a viral kinase and then converted to the diphosphate forms by cellular GMPK (Sekulic et al. 2002).

GMPK is a member of the family of ATP:NMP phosphoryltransferases (nucleoside monophosphate kinases; NMP kinases, or NMPKs). NMP kinases of different species (E. coli, yeast, mouse, and human) have been characterized for their structure and function showing similarities to the class of nucleoside kinases (e.g., human deoxycytidine kinase). NMP kinases and nucleoside kinases share distinctive structural features: the phosphate-binding loop (P-loop) as a common motif found in most ATP- and GTPbinding proteins, the NMP-binding domain (NMP-BD), a LID domain, and a CORE domain, which are connected by dynamic hinges (Hazra et al. 2010; Lavie et al. 1998a, b; Ostermann et al. 2000) (Fig. 1). NMP kinases are bisubstrate enzymes that bind both substrates simultaneously to catalyze phosphoryl transfer. These kinases exist in different conformations: without ligands (apo-form), NMP-bound form, ATP-bound form, and a ternary complex with bound NMP and ATP (closed form). The binding of substrates to NMP kinases reassembles their active centers resulting in large domain movements (Muller-Dieckmann and Schulz 1995). Large conformational changes in NMP kinases were first observed in adenylate kinases (AMP kinases; AKs) (Gerstein et al. 1993; Schulz et al. 1990). Structural analysis of various homologous forms of AKs showed that both ATP and AMP induce substantial conformational changes upon binding to these enzymes. The binding of AMP resulted in the closure of the NMP-BD domain, whereas the binding of ATP caused the closure of the LID domain (Yan and Tsai 1999). The analysis of 17 crystal structures from the NMP kinase family confirmed the existence of these large conformational changes, which were mainly attributed to rigid-body movements of the LID and NMP-BD with respect to the CORE domain (Vonrhein et al. 1995). Similar observations were made for yeast GMPK (yGMPK). Large movements of the GMP-binding 
Fig. 1 The Swiss homology model for hGMPK was constructed by using the crystal structure of mGMPK's closed conformation (residues 5-194 of $197 \mathrm{aa}$ ) as template [ $p d b 1 L V G$ (Sekulic et al. 2002)]. The three major structural regions present in hGMPK are designated and color-coded as the NMP-binding domain (NMP-BD) (blue), the CORE (red), and the LID (green). These structural regions in hGMPK (residues 5-194 of $197 \mathrm{aa}$ ) are interconnected by four hinges (yellow). hGMPK substrates, GMP (blue square), which bind to NMP-BD and substrate ATP (green triangle), which bind to LID in GMPK are schematically presented

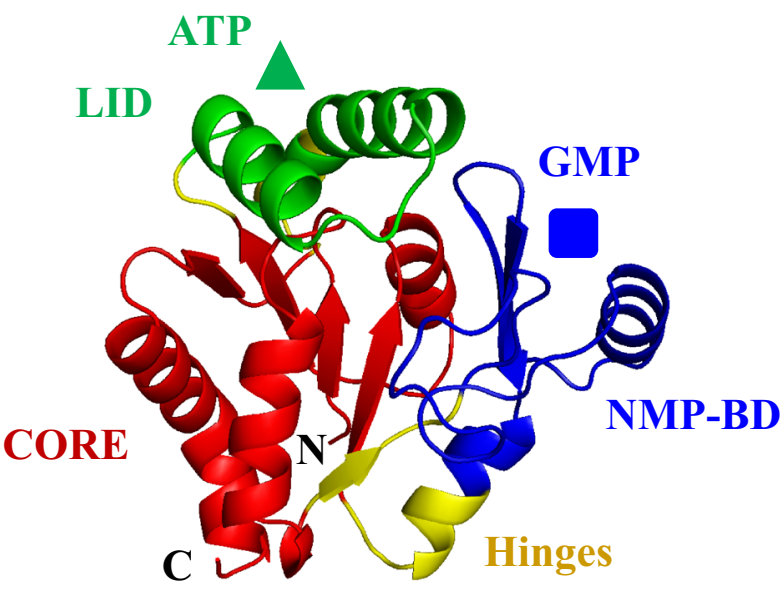

5 \begin{tabular}{l} 
RPVVLSGPSGAGKSTLLKRLLQEHSGIFGFSVSHTTRNPRPGEENGKDYYFVTREVMQRDIAAG \\
DFIEHAEFSGLYGTSKVAVQAVOAMNRICVLDVDLQGVRNIKATDLRPIYISVQPPSLHVLEQ \\
RLRQRNTETEESLVKRLAAAQADMESSKEPGLFDVVIINDSLDQAYAELKEALSEEIKKAQR \\
\hline
\end{tabular} domain and smaller but significant movements of the LID domain were reported by comparing the open yGMPK (apo) conformation and the partially closed yGMPK (GMP) conformation. Also, the open yGMPK conformation was found to be more open than its substrate-bound partially closed conformation (Blaszczyk et al. 2001).

Despite their important catalytic and therapeutic roles, the only structural information available for mammalian GMPKs is the crystal structure of the fully closed form of mouse GMPK in complex with one substrate and one product molecule (mGMPK (GMP + ADP), pdb $1 L V G$ ) (Sekulic et al. 2002). The lack of structural and dynamical information for hGMPK could be due to the purification of recombinant hGMPK in the inactive form and the failures of crystallization (Ardiani et al. 2009; Brady et al. 1996; Sekulic et al. 2002). Small-angle X-ray scattering (SAXS) only requires small amounts of purified hGMPK in solution to provide global structural information, assess structural changes, and derive the three-dimensional surface reconstructions of hGMPK in the absence and presence of its substrates and analogs (Jacques and Trewhella 2010; Jain et al. 2013; Koch et al. 2003; Mallik et al. 2012; Petri et al. 2011, 2012; Putnam et al. 2007; Solanki et al. 2014; Taylor et al. 2012; Tuukkanen and Svergun 2014). We succeeded in purifying the active recombinant hGMPK, which was used to obtain insights into between open/closed hGMPK conformations. SAXS measurements were performed at a home X-ray source and at a third-generation X-ray synchrotron. In these studies, the open hGMPK conformation (no ligand) was compared with three different completely closed hGMPK conformations [GMP + AMPPNP (1),
Ap5G (2), and GMP + ADP (3)]. We also evaluated the reconstructed SAXS models (ab initio) of different hGMPK conformations with the crystal structure of its murine homologue, the fully closed form of mGMPK (Sekulic et al. 2002). Different hGMPK conformations were also investigated with circular dichroism to confirm small structural differences observed with SAXS. These investigations may explain the mode of interaction of anticancer and antiviral therapeutics with hGMPK, which is a critical enzyme for the metabolic activation of certain nucleoside analogs.

\section{Materials and methods}

\section{Cloning, expression, and purification of human guanylate kinase}

The 591-bp open reading frame (ORF) of hGMPK (UniProt entry Q16774, also called GMP kinase, GUK1, or GMK), was amplified via polymerase chain reaction (PCR) using the DNA template reported previously (Kuhlendahl et al. 1998; Kumar et al. 2000; Prinz et al. 1999). The final construct includes an N-terminal hexa-histidine tag followed by the SUMO (small ubiquitin-related modifier; SUMO family protein SMT3 of 101 residues)-tag, which was used to improve heterologous protein solubility and stability (Panavas et al. 2009). The detailed hGMPK cloning and purification procedure is described in the supplementary information. For overproduction of the enzyme, the E. coli expression strain BL21-(DE3)-pLysS carrying the hGMPK plasmid was used to inoculate 11 of lactose-containing 
auto-inducing medium. hGMPK was obtained as a catalytically active monomer upon applying different purification steps. The monomer peak of hGMPK was pooled, concentrated to $38 \mathrm{mg} / \mathrm{ml}$, aliquoted, and then stored at $-80{ }^{\circ} \mathrm{C}$. The Bradford dye-binding assay was used to determine the hGMPK concentration.

\section{Enzyme activity assay}

The enzymatic activity of hGMPK was determined by a standard NADH-dependent lactate dehydrogenase/pyruvate kinase-coupled assay using a JASCO V-650 UV-Vis spectrophotometer (Konrad 1992). All measurements were performed at $25{ }^{\circ} \mathrm{C}$ in the assay buffer, which consisted of $100 \mathrm{mM}$ Tris- $\mathrm{HCl}, \mathrm{pH} 7.5,100 \mathrm{mM} \mathrm{KCl}$, and $10 \mathrm{mM}$ $\mathrm{MgCl}_{2}$. The final hGMPK concentration in the assay was $18 \mathrm{nM}$.

\section{Home source SAXS data acquisition}

SAXS data was collected at the home-built SAXS apparatus (Quevedo et al. 2008) to check the effect of various hGMPK concentrations. The hGMPK samples and corresponding buffers in different experimental conditions were exposed and measured under stationary conditions in the assembled quartz capillary flow cell with $1.0 \mathrm{~mm}$ diameter. Potential radiation damage in the measured samples was checked with SDS-PAGE and the kinase activity assay. In this way, we verified that the enzyme did not suffer from radiation damage during $\mathrm{X}$-ray exposures.

\section{Synchrotron SAXS data acquisition}

hGMPK samples and their matching buffers under different experimental conditions were filled in separate $1.0-\mathrm{mm}$ diameter borosilicate capillaries and sealed with capillary wax for SAXS measurements. SAXS data was collected at the coherent SAXS beamline X12SA, Swiss Light Source (SLS) at the Paul Scherrer Institute (PSI, Switzerland). The photon flux was $\sim 10^{12}$ photons $/ \mathrm{s}$ at the sample position. The photon energy was $12.4 \mathrm{keV}$, which corresponds to a wavelength of $1.0 \AA$. X-ray images were acquired with an integration time of $100 \mathrm{~ms}$ per image.

The X-ray spot size on the sample was $100 \times 100 \mu \mathrm{m}$ and the X-ray beam was focused on the detector. SAXS images were acquired using a PILATUS $2 \mathrm{M}$ detector at a sample-detector distance of $\sim 7 \mathrm{~m}$. Images were acquired at three different spots on the capillary; these observation spots were $1.0 \mathrm{~mm}$ apart and were centered across the capillary.

\section{SAXS data processing and analysis}

Scattering curves were obtained from the raw PILATUS detector images using MATLAB (R2012a) and further processed with Origin (8.6) and ATSAS (2.4) software (Konarev et al. 2006; Petoukhov et al. 2012). hGMPK scattering curves $I(q)$ vs. $q$ were plotted after the subtraction of their respective buffer from the hGMPK samples as a function of momentum transfer $q\left(\AA^{-1}\right)$, which is given by:

$q=\frac{4 \pi \sin \theta}{\lambda}$,

where $2 \theta$ is the scattering angle and $\lambda$ is the $\mathrm{X}$-ray wavelength in $\AA$ (Glatter and Kratky 1982; Guinier and Fournet 1955).

The SAXS scattering curves were then analyzed with different parameters. The Guinier approximation for a monodisperse sample of globular protein can be used to describe the X-ray scattering at low $q$ as:

$\ln [I(q)]=\ln [I(0)]-\left(\frac{R_{\mathrm{g}}^{2}}{3}\right) \times\left(q^{2}\right)$,

where $R_{\mathrm{g}}$ is the radius of gyration and $I(0)$ is the forward scattered intensity (Glatter and Kratky 1982; Guinier and Fournet 1955). The Guinier approximation is valid for the $q R_{\mathrm{g}} \leq 1.3$ region. $R_{\mathrm{g}}$ is essentially the second moment of the distribution of shape and size of proteins about the mean and its calculation requires electron density rather than mass as a weighing factor. For this $q$ range, the Guinier plot will give a straight line from which $R_{\mathrm{g}}$ and $I(0)$ values are extracted. $R_{\mathrm{g}}$ values were determined by various procedures including manual Guinier plot (not shown), PRIMUS and GNOM (Konarev et al. 2003; Svergun et al. $1995)$. The $I(0)$ value is directly proportional to the multiplication of molar protein concentrations and molecular mass of the scattering hGMPK molecule. $I(0)$ was approximated by the extrapolation of SAXS intensity to $q=0$. The Kratky plots $\left(I(q) \times q^{2}\right.$ vs. $\left.q\right)$ were then used to assess the globular or Gaussian chain-like nature of hGMPK under different experimental conditions.

ATSAS suite was used to reconstruct and analyze the three-dimensional SAXS models. The detailed procedure is presented in the supplementary information. The inertial axes of the SAXS data-based averaged hGMPK models were then overlaid to different crystal structures or to the different SAXS models by the SUPCOMB13 software (Kozin and Svergun 2001). The open-source software PyMOL was used for graphical analysis and for figure preparation. 


\section{CD measurements of different hGMPK conformations}

CD measurements were performed to confirm the observed size and shape changes in different hGMPK conformations with SAXS. hGMPK concentration was $80 \mu \mathrm{M}$. The buffer was $10 \mathrm{mM}$ potassium phosphate $\mathrm{pH}=7.5,2 \mathrm{mM} \mathrm{MgSO}_{4}$, and $0.5 \mathrm{mM}$ Tris (2-carboxyethyl)phosphine (TCEP). Substrate (GMP + AMP-PNP and GMP + ADP) concentration was $1 \mathrm{mM}$ in hGMPK samples and buffer. The bi-substrate analog Ap5G was used at $100 \mu \mathrm{M}$ concentration.

CD spectra were measured separately for hGMPK samples and respective buffers at $25{ }^{\circ} \mathrm{C}$ between 180 and $300 \mathrm{~nm}$. Five scans were collected at $1 \mathrm{~s} /$ measurement and then averaged. The averaged CD spectra for an hGMPK conformation and its respective buffer was subtracted and then smoothed (window size $=3$ ). The CD spectra were finally plotted with the help of Origin 8.6.

\section{Results and discussion}

Human guanylate kinase, hGMPK, was expressed in $E$. coli, and the histidine-tagged protein $(\sim 22 \mathrm{kDa})$ was purified via nickel agarose-affinity chromatography and sizeexclusion chromatography in successive steps (Fig. 2a). The purified recombinant hGMPK protein was catalytically active as determined in the NADH-dependent enzymecoupled assay and yielded the kinetic parameters shown in Table 1 . The activity of recombinant hGMPK presented in this work is in contrast to previous reports where hGMPK was found inactive (Ardiani et al. 2009; Brady et al. 1996).

The radius of gyration $\left(R_{\mathrm{g}}\right)$ and forward scattered scattering $(I(0))$ of hGMPK remained unchanged for increasing concentrations $(6.5-26.5 \mathrm{mg} / \mathrm{ml})$ in the home SAXS measurements (data not shown). Also, the hGMPK activity

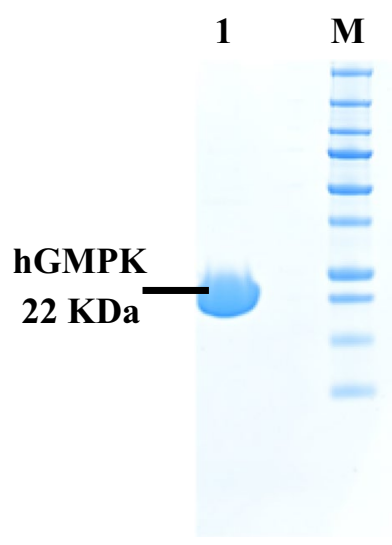

Fig. 2 Purified recombinant hGMPK is catalytically active. Bacterially produced hGMPK was purified by affinity and gel filtration chromatography in two successive steps. Purified hGMPK is a single band on $12 \%$ SDS-PAGE
Table 1 Kinetic parameters obtained from the steady-state kinetic assay measuring hGMPK activity $\left(T=25^{\circ} \mathrm{C}\right)$. The ATP:GMP phosphoryl transferase activity of purified recombinant hGMPK was determined by using the NADH-dependent enzyme-coupled assay

\begin{tabular}{llll}
\hline Substrate & $k_{\mathrm{cat}}\left(\mathrm{s}^{-1}\right)$ & $k_{m}(\mu \mathrm{M})$ & $k_{\mathrm{cat}} / K_{m}\left(\mathrm{M}^{-1} \mathrm{~s}^{-1}\right)$ \\
\hline GMP & 79 & 25 & $3.2 \times 10^{6}$ \\
Mg-ATP & 79 & 95 & $0.8 \times 10^{6}$ \\
\hline
\end{tabular}

did not decrease after X-ray exposure during SAXS measurements (data not shown). In the following step, different hGMPK conformations in the presence and absence of substrates and analogs were measured at the third-generation cSAXS beamline: No ligand (open form), GMP + AMPPNP (completely closed form 1), Ap5G (completely closed form 2), and GMP + ADP (completely closed form 3). The SAXS measurements were performed for corresponding buffer solutions as well. SAXS measurements of different hGMPK conformations along with buffer were free from radiation damage. This was checked by plotting integral X-ray intensity of consecutive scattering images at an observation spot with respect to the X-ray exposure time (Fig. S2, ESM). The buffer-subtracted SAXS intensity profiles of different hGMPK conformations were plotted and analyzed with various SAXS parameters to evaluate structural changes in different hGMPK conformations (Fig. 3a). The bell-shaped Kratky profiles depict a globular nature for all measured hGMPK conformations (Fig. 3b).

The unliganded form of hGMPK was found to be monomeric by comparing its elution profile with standard proteins by size-exclusion chromatography (Fig. S1 and Table S1, ESM). Open and completely closed hGMPK conformations (1-3) have identical $I(0)$ values (Table 2). This implies that hGMPK is a monomer in all investigated conformations and the binding of substrates and analogs did not cause its oligomerization. Different slopes of manual Guinier plots for hGMPK conformations indicate variation in the hGMPK size (Fig. 3c). The size $\left(R_{\mathrm{g}}\right)$ of different hGMPK conformations was calculated by pair distance distribution curves (Table 2). The open hGMPK conformation is bigger in size $\left(R_{\mathrm{g}} \sim 20.1( \pm 0.04) \AA\right)$ than the completely closed hGMPK conformations: GMP + AMPPNP (1, $\left.R_{\mathrm{g}} \sim 18.4( \pm 0.04) \AA\right), \mathrm{Ap} 5 \mathrm{G}\left(2, R_{\mathrm{g}} \sim 18.1( \pm 0.03) \AA\right)$ and GMP + ADP ( $\left.3, R_{\mathrm{g}} \sim 18.4( \pm 0.04) \AA\right)$. In tendency, the completely closed hGMPK conformations (1-3) get compact in comparison to the open hGMPK conformation. The maximum dimensions $D_{\max }$ for different hGMPK conformations were also calculated with the pair distance distribution curves (Fig. 3d; Table 2). The maximum dimensions of open and completely closed hGMPK conformations (1-3) are similar $\left(D_{\max } \sim 50 \AA\right.$ ) (Table 2).

The overall fold of hGMPK is very similar to that of other members of the NMP-kinase family, in particular to 
Fig. 3 SAXS data analysis for open and completely closed hGMPK conformations. a SAXS intensity curves. b Kratky profiles. c Guinier plots. d Computed pair distance distributions
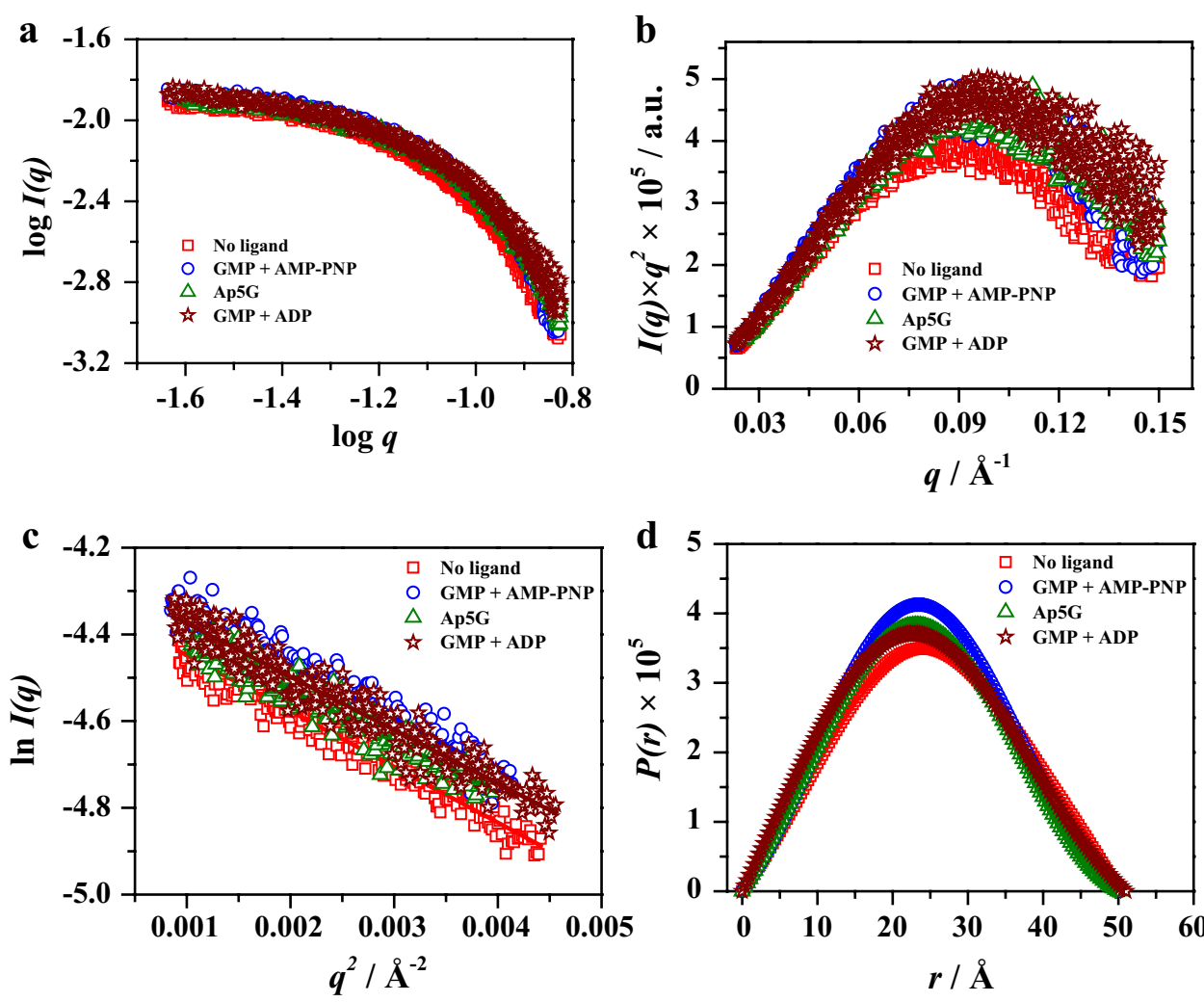

Table 2 Structural parameters for different hGMPK conformations as derived with SAXS measurements

\begin{tabular}{lllll}
\hline hGMPK sample & Conformation & $I(0) \times 10^{-2}$ & $R_{\mathrm{g}}(\AA)$ & $D_{\max }(\AA)$ \\
\hline Apo-form & Open & 1.3 & $20.1( \pm 0.04) 50.0$ \\
$\begin{array}{l}\text { GMP + AMP- } \\
\text { PNP }\end{array}$ & $\begin{array}{c}\text { Completely } \\
\text { closed 1 }\end{array}$ & 1.4 & $18.4( \pm 0.04) 50.0$ \\
Ap5G & $\begin{array}{c}\text { Completely } \\
\text { closed 2 }\end{array}$ & 1.4 & $18.1( \pm 0.03) 50.0$ \\
GMP + ADP & $\begin{array}{c}\text { Completely } \\
\text { closed 3 }\end{array}$ & 1.4 & $18.4( \pm 0.04) 51.0$ \\
\hline
\end{tabular}

mGMPK and yGMPK (Blaszczyk et al. 2001; Kandeel and Kitade 2011; Sekulic et al. 2002). The 197-amino-acid-long hGMPK is just one amino acid shorter at the C-terminus than the closely related mGMPK, with which it has high amino acid sequence identity (88\%). The crystal structures of open yGMPK [apo, pdb 1EX6 (Blaszczyk et al. 2001)] and partially closed yGMPK [GMP, pdb 1EX7 (Blaszczyk et al. 2001)] are also available. All available GMPK structures share three major structural domains: the NMP-BD, a CORE domain, and a LID domain, which are interconnected by four dynamic hinges. In its apo-form (yGMPK without ligand), the NMP-BD and the LID domain are farthest apart, and therefore the open form of yGMPK is more extended than the partially closed form of yGMPK (Blaszczyk et al. 2001). The binding of GMP to the NMPBD in yGMPK (in complex with GMP) causes a significant movement of the NMP-BD towards the LID domain with comparatively smaller movement of the LID domain in the same direction. Therefore, GMP binding brings the NMP-BD and the LID domain closer to each other resulting in a compaction of the partially closed yGMPK (GMPcomplexed) as compared to its open yGMPK (apo) form (Blaszczyk et al. 2001). The simultaneous binding of both substrates (GMP + ADP) in mGMPK brings the NMP-BD and LID domain closer together than in the partially closed yGMPK (GMP) conformation (Sekulic et al. 2002). Therefore, the fully closed conformation of GMPK is the most compact conformation (Sekulic et al. 2002). Also, the open form of yGMPK is the most flexible conformation, while the fully closed hGMPK form is the most rigid conformation (Blaszczyk et al. 2001; Sekulic et al. 2002).

Changes in the global structure of hGMPK from open to completely closed conformation were investigated by reconstructing low resolution SAXS models within the shape constraints computed with $P(r)$ function analysis. Structural interpretation for each hGMPK conformation was performed with 10-15 reconstructed SAXS models ( $p d b$ files) having low $\chi^{2}$ values between experimental 
scattering intensity and computed scattering intensity for the GASBOR models $\left(\chi^{2}=1.6-2.6\right)$ or DAMMIN models $\left(\chi^{2}=1.5-2.2\right)$ (Table S2, ESM). The SAXS models generated with GASBOR or DAMMIN programme were averaged separately by DAMAVER (Fig. S2, ESM). The alignment and averaging procedure for the reconstructed SAXS models was scrutinized with normalized spatial discrepancy (NSD) values. DAMMIN and GASBOR models with higher NSD values (NSD $>$ mean $+2 \times$ variation) were discarded (Table S2, ESM). To identify the structural changes in the hGMPK molecule, the averaged SAXS models of different hGMPK conformations were overlaid on the crystal structure of the fully closed mGMPK form [pdb ILVG (Sekulic et al. 2002)] (Fig. 4, Tables S3, S4, ESM). With respect to the NSD values, the overlays were sufficient and revealed compactness from open to completely closed hGMPK conformations (Fig. 4, Tables S3, S4, ESM).

A

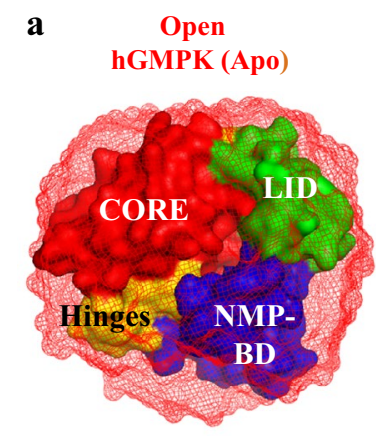

vs. Completely closed mGMPK (GMP + ADP) crystal
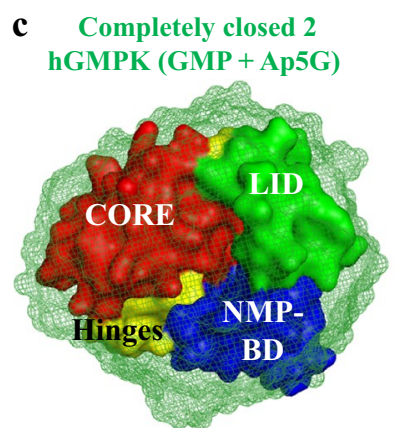

vs. Completely closed mGMPK (GMP + ADP) crystal
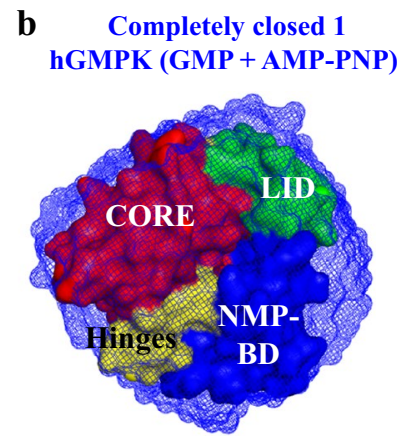

vs. Completely closed mGMPK (GMP + ADP) crystal

Completely closed 3
hGMPK (GMP + ADP)

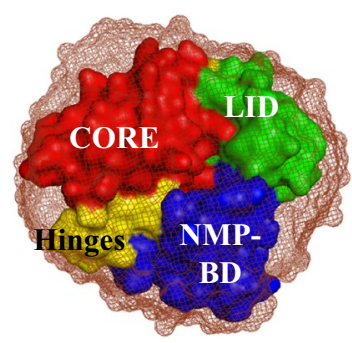

vs. Completely closed mGMPK (GMP + ADP) crystal
B

a

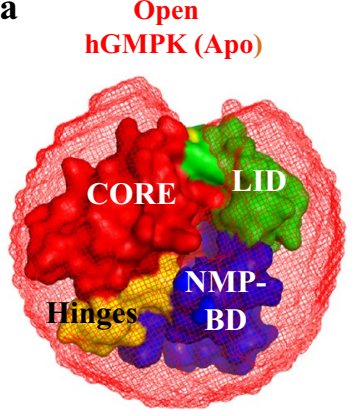

vs. Completely closed mGMPK (GMP + ADP) crystal

c Completely closed 2 hGMPK (GMP + Ap5G)

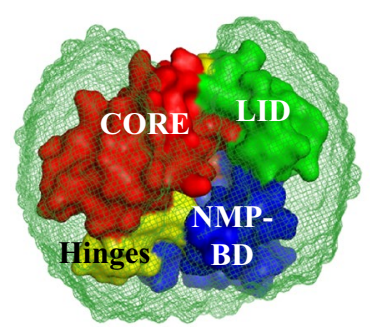

vs. Completely closed mGMPK (GMP + ADP) crystal b Completely closed 1 hGMPK (GMP + AMP-PNP)

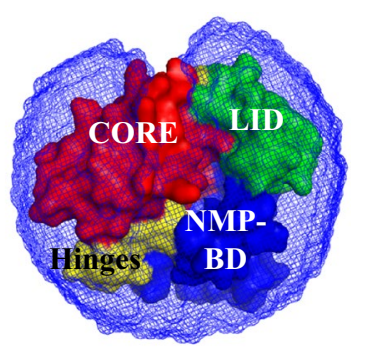

vs. Completely closed mGMPK (GMP + ADP) crystal

d Completely closed 3 hGMPK (GMP + ADP)

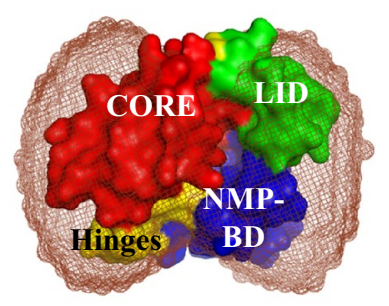

vs. Completely closed mGMPK (GMP + ADP) crystal

Fig. 4 Overlay of reconstructed hGMPK SAXS models (mesh representation) on the crystal structure of the completely closed mGMPK conformation (GMP + ADP, $p d b$ 1LVG (Sekulic et al. 2002), surface representation). GASBOR (A) and DAMMIN (B) SAXS models of different hGMPK conformations are compared separately. a Open hGMPK conformation (apo-form). b Completely closed hGMPK

conformation 1 (GMP + AMP-PNP). c Completely closed hGMPK conformation 2 (Ap5G). d Completely closed hGMPK conformation 3 (GMP + ADP). Overlay was done with SUPCOMB13 and the resulting NSD values are shown in Table S4, ESM. Different structural regions in the crystal structure of completely closed mGMPK (NMP-BD, CORE, and LID) and hinges are color-coded 
Three-dimensional reconstructions are globular for the open and completely closed hGMPK conformations (Fig. 4). There are small but distinguishable differences in shape between open and completely closed hGMPK conformations. The open hGMPK conformation is characteristic of the unliganded enzyme. The binding of GMP (substrate) to the NMP-BD and AMP-PNP (ATP analog) to the LID domain induces the completely closed hGMPK conformation 1. The binding of the bi-substrate analog Ap5G to NMP-BD and LID domain induces the completely closed hGMPK conformation 2. The binding of GMP (substrate) to the NMP-BD and ADP (reaction product) to the LID domain induces the completely closed hGMPK conformation 3. The completely closed hGMPK conformations are smaller in size than those of the open hGMPK conformation (Fig. 4).

The compaction observed from open to completely closed hGMPK conformations was compared with small differences in the size between crystal structures of open yeast conformation ( $p d b \quad 1 L V G$ ), partially closed conformation ( $p d b$ 1EX6) and completely closed mGMPK conformation ( $p d b$ 1EX7). The open to completely closed hGMPK conformation follows the trend observed in the size reduction from open yeast conformation $\left(R_{\mathrm{g}} \sim 18.8 \AA\right)$ to the partially closed yeast GMPK conformation $\left(R_{\mathrm{g}} \sim 17.8 \AA\right)$ and to the completely closed mGMPK conformation $\left(R_{\mathrm{g}} \sim 17.2\right.$ $\AA$ ) (Tables S5, ESM). One way to explain the compaction between open hGMPK and completely closed hGMPK could be the closeness of the NMP-BD and ATP-binding domains in comparison to the open hGMPK conformation. The observed structural changes in the size and shape of open and closed hGMPK conformations were confirmed by circular dichroism (CD) spectroscopy. CD spectroscopy is a valuable technique for the investigation of structural changes in proteins caused by the binding of ligands (Fasshauer et al. 2002; Greenfield 2006; Petri et al. 2012). The differences in secondary structures from open to completely closed hGMPK conformations (1-3) observed with CD spectra are small and present at 210-240 nm (peptide bonds) and 260-300 nm (tertiary structure) (Fig. 5). Hence, the compaction in hGMPK structure during catalytic reaction (open to completely closed) could result from a change in the secondary structure content of hGMPK.

In summary, our SAXS analyses have tracked the missing structural differences in the size and shape of open and completely closed hGMPK conformations. The opento-close conformational transition of hGMPK brought about by the sequential binding of its substrates supports the induced fit enzymatic mechanism. The good fitting of the completely closed mGMPK crystal structure and completely closed hGMPK SAXS models indicates the conservation of the 3D-fold of the guanylate kinase domain in these two enzymes.

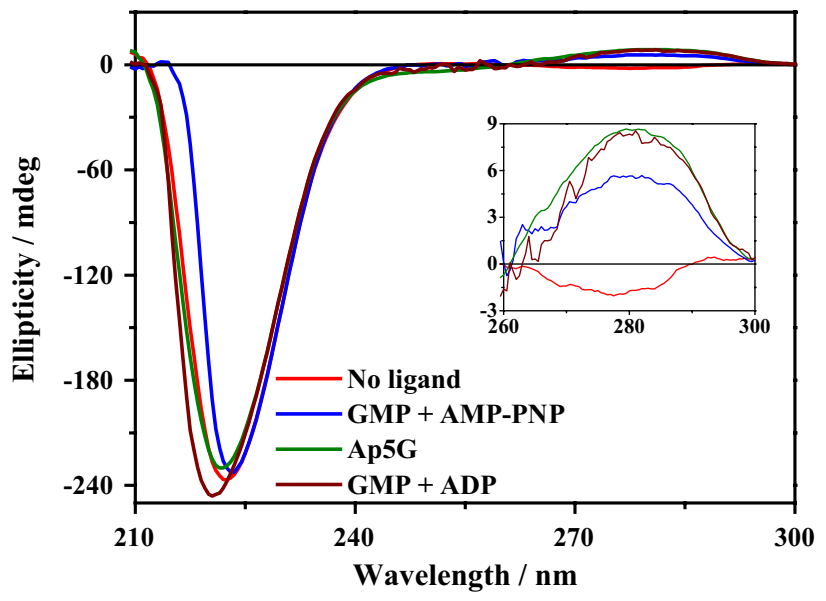

Fig. 5 CD spectrums of different hGMPK conformations

Acknowledgments SAXS experiments have been performed at the cSAXS beamline (PSI, Switzerland). We thank Gerhard Busse and the Machine shop (MPI-BPC) for excellent technical assistance at the home SAXS apparatus. We acknowledge funding by the Max Planck Society. Nazimuddin Khan was supported by a DAAD scholarship. The work was supported by project B10 of SFB 755 "Nanoscale Photonic Imaging”.

Open Access This article is distributed under the terms of the Creative Commons Attribution 4.0 International License (http://creativecommons.org/licenses/by/4.0/), which permits unrestricted use, distribution, and reproduction in any medium, provided you give appropriate credit to the original author(s) and the source, provide a link to the Creative Commons license, and indicate if changes were made.

\section{References}

Ardiani A, Goyke A, Black ME (2009) Mutations at serine 37 in mouse guanylate kinase confer resistance to 6-thioguanine. Protein Eng Des Sel PEDS 22:225-232

Beck BJ, Huelsmeyer M, Paul S, Downs DM (2003) A mutation in the essential gene gmk (encoding guanlyate kinase) generates a requirement for adenine at low temperature in Salmonella enterica. J Bacteriol 185:6732-6735

Blaszczyk J, Li Y, Yan H, Ji X (2001) Crystal structure of unligated guanylate kinase from yeast reveals GMP-induced conformational changes. J Mol Biol 307:247-257

Brady WA, Kokoris MS, Fitzgibbon M, Black ME (1996) Cloning, characterization, and modeling of mouse and human guanylate kinases. J Biol Chem 271:16734-16740

Choi B, Zocchi G (2007) Guanylate kinase, induced fit, and the allosteric spring probe. Biophys J 92:1651-1658

Fasshauer D, Antonin W, Subramaniam V, Jahn R (2002) SNARE assembly and disassembly exhibit a pronounced hysteresis. Nature structural biology 9:144-151

Gerstein M, Schulz G, Chothia C (1993) Domain closure in adenylate kinase. Joints on either side of two helices close like neighboring fingers. J Mol Biol 229:494-501

Glatter O, Kratky O (1982) Small-angle X-ray scattering. Academic Press, London 
Greenfield NJ (2006) Using circular dichroism spectra to estimate protein secondary structure. Nat Protoc 1:2876-2890

Guinier A, Fournet G (1955) Small-angle scattering of X-rays. Wiley, New York

Hall SW, Kuhn H (1986) Purification and properties of guanylate kinase from bovine retinas and rod outer segments. Eur J Biochem/FEBS 161:551-556

Hazra S, Ort S, Konrad M, Lavie A (2010) Structural and kinetic characterization of human deoxycytidine kinase variants able to phosphorylate 5-substituted deoxycytidine and thymidine analogues. Biochemistry 49:6784-6790

Jacques DA, Trewhella J (2010) Small-angle scattering for structural biology-expanding the frontier while avoiding the pitfalls. Protein Sci 19:642-657

Jain R, Petri M, Kirschbaum S, Feindt H, Steltenkamp S, Sonnenkalb S, Becker S, Griesinger C, Menzel A, Burg TP, Techert S (2013) $\mathrm{X}$-ray scattering experiments with high-flux X-ray source coupled rapid mixing microchannel device and their potential for high-flux neutron scattering investigations. Eur Phys J E Soft Matter 36:109

Kandeel M, Kitade Y (2011) Binding dynamics and energetic insight into the molecular forces driving nucleotide binding by guanylate kinase. J Mole Recognit JMR 24:322-332

Karran P (2006) Thiopurines, DNA damage, DNA repair and therapyrelated cancer. Br Med Bull 79-80:153-170

Karran P, Attard N (2008) Thiopurines in current medical practice: molecular mechanisms and contributions to therapy-related cancer. Nat Rev Cancer 8:24-36

Koch MH, Vachette P, Svergun DI (2003) Small-angle scattering: a view on the properties, structures and structural changes of biological macromolecules in solution. Q Rev Biophys 36:147-227

Konarev PV, Volkov VV, Sokolova AV, Koch MHJ, Svergun DI (2003) PRIMUS: a windows PC-based system for small-angle scattering data analysis. J Appl Crystallogr 36:1277-1282

Konarev PV, Petoukhov MV, Volkov VV, Svergun DI (2006) ATSAS 2.1, a program package for small-angle scattering data analysis. J Appl Crystallogr 39:277-286

Konrad M (1992) Cloning and expression of the essential gene for guanylate kinase from yeast. J Biol Chem 267:25652-25655

Kozin MB, Svergun DI (2001) Automated matching of high- and lowresolution structural models. J Appl Crystallogr 34:33-41

Kuhlendahl S, Spangenberg O, Konrad M, Kim E, Garner CC (1998) Functional analysis of the guanylate kinase-like domain in the synapse-associated protein SAP97. Eur J Biochem/FEBS 252:305-313

Kumar V, Spangenberg O, Konrad M (2000) Cloning of the guanylate kinase homologues AGK-1 and AGK-2 from Arabidopsis thaliana and characterization of AGK-1. Eur J Biochem/FEBS 267:606-615

Lavie A, Konrad M, Brundiers R, Goody RS, Schlichting I, Reinstein J (1998a) Crystal structure of yeast thymidylate kinase complexed with the bisubstrate inhibitor P1-(5'-adenosyl) P5-(5'-thymidyl) pentaphosphate (TP5A) at 2.0 A resolution: implications for catalysis and AZT activation. Biochemistry 37:3677-3686

Lavie A, Ostermann N, Brundiers R, Goody RS, Reinstein J, Konrad M, Schlichting I (1998b) Structural basis for efficient phosphorylation of $3^{\prime}$-azidothymidine monophosphate by Escherichia coli thymidylate kinase. Proc Natl Acad Sci 95:14045-14050

Mallik L, Arif E, Sharma P, Rathore YS, Wong HN, Holzman LB, Ashish, Nihalani D (2012) Solution structure analysis of cytoplasmic domain of podocyte protein Neph1 using small/wide angle X-ray scattering (SWAXS). J Biol Chem 287:9441-9453
Muller-Dieckmann HJ, Schulz GE (1995) Substrate specificity and assembly of the catalytic center derived from two structures of ligated uridylate kinase. J Mol Biol 246:522-530

Ostermann N, Schlichting I, Brundiers R, Konrad M, Reinstein J, Veit T, Goody RS, Lavie A (2000) Insights into the phosphoryltransfer mechanism of human thymidylate kinase gained from crystal structures of enzyme complexes along the reaction coordinate. Structure (London, England : 1993) 8:629-642

Panavas T, Sanders C, Butt TR (2009) SUMO fusion technology for enhanced protein production in prokaryotic and eukaryotic expression systems. Method Mol Biol (Clifton, NJ) 497:303-317

Petoukhov MV, Franke D, Shkumatov AV, Tria G, Kikhney AG, Gajda M, Gorba C, Mertens HDT, Konarev PV, Svergun DI (2012) New developments in the ATSAS program package for smallangle scattering data analysis. J Appl Crystallogr 45:342-350

Petri M, Menzel A, Bunk O, Busse G, Techert S (2011) Concentration effects on the dynamics of liquid crystalline self-assembly: time-resolved X-ray scattering studies. J Phys Chem A 115:2176-2183

Petri M, Frey S, Menzel A, Gorlich D, Techert S (2012) Structural characterization of nanoscale meshworks within a nucleoporin FG hydrogel. Biomacromolecules 13:1882-1889

Prinz H, Lavie A, Scheidig AJ, Spangenberg O, Konrad M (1999) Binding of nucleotides to guanylate kinase, p21(ras), and nucleoside-diphosphate kinase studied by nano-electrospray mass spectrometry. J Biol Chem 274:35337-35342

Putnam CD, Hammel M, Hura GL, Tainer JA (2007) X-ray solution scattering (SAXS) combined with crystallography and computation: defining accurate macromolecular structures, conformations and assemblies in solution. Q Rev Biophys 40:191-285

Quevedo W, Petri M, Techert S (2008) Home-based time-resolved photo small-angle X-ray diffraction and its applications. Zeitschrift für Kristallographie-Cryst Mater 223:322-328

Schulz GE, Muller CW, Diederichs K (1990) Induced-fit movements in adenylate kinases. J Mol Biol 213:627-630

Sekulic N, Shuvalova L, Spangenberg O, Konrad M, Lavie A (2002) Structural characterization of the closed conformation of mouse guanylate kinase. J Biol Chem 277:30236-30243

Solanki AK, Rathore YS, Badmalia MD, Dhoke RR, Nath SK, Nihalani D, Ashish (2014) Global shape and ligand binding efficiency of the HIV-1-neutralizing antibodies differ from those of antibodies that cannot neutralize HIV-1. J Biol Chem 289:34780-34800

Svergun D, Barberato C, Koch MHJ (1995) CRYSOL-a program to evaluate X-ray solution scattering of biological macromolecules from atomic coordinates. J Appl Crystallogr 28:768-773

Taylor JE, Chow JY, Jeffries CM, Kwan AH, Duff AP, Hamilton WA, Trewhella J (2012) Calmodulin binds a highly extended HIV-1 MA protein that refolds upon its release. Biophys J 103:541-549

Tuukkanen AT, Svergun DI (2014) Weak protein-ligand interactions studied by small-angle X-ray scattering. FEBS J 281:1974-1987

Vonrhein C, Schlauderer GJ, Schulz GE (1995) Movie of the structural changes during a catalytic cycle of nucleoside monophosphate kinases. Structure (London, England: 1993) 3:483-490

Yan H, Tsai MD (1999) Nucleoside monophosphate kinases: structure, mechanism, and substrate specificity. Adv Enzymol Relat Areas Mol Biol 73:103-134

Zhang F, Fu L, Wang Y (2013) 6-thioguanine induces mitochondrial dysfunction and oxidative DNA damage in acute lymphoblastic leukemia cells. Mol Cell Proteom MCP 12:3803-3811 